\title{
THEORY AND DESIGN OF UNIFORM CONCENTRIC CIRCULAR ARRAYS WITH FREQUENCY INVARIANT CHARACTERISTICS
}

\author{
S. C. Chan and H. H. Chen \\ Department of Electrical and Electronic Engineering \\ The University of Hong Kong, Pokfulam Road, Hong Kong
}

\begin{abstract}
This paper proposes a new digital beamformer for uniform concentric circular array (UCCA) having nearly frequency invariant (FI) characteristics. The basic principle is to transform the received signals to the phase mode and remove the frequency dependency of the individual phase mode through the use of a digital beamforming network. The far field pattern of the array is determined by a set of weights and it is approximately invariant over a wide range of frequencies. Compared with FI uniform circular array (UCA), FI UCCAs are able to achieve a wider bandwidth. Design examples are given to demonstrate the principle of the proposed UCCA-FIB and its application to broadband DOA estimation of coherent sources.
\end{abstract}

\section{INTRODUCTION}

Wideband beamforming using sensor arrays is an effective method for suppressing interference whose angles of arrival are different from the desired looking direction. They find important applications in radio communications, sonar, radar, and acoustics [1-3]. Traditional adaptive wideband beamformer usually employs tapped-delay line with adaptive coefficients to generate appropriate beam patterns for interference suppression. This usually requires considerable number of adaptive coefficients resulting in rather long convergence time and high implementation complexity. This can be remedied by using subband decomposition technique, partial adaptation or using frequency invariant beamformers (FIB) [4-6,7,9]. In FIB, a beam-forming network is used to generate beam pattern with approximately frequency invariant (FI) characteristics over the frequency band of interest. They can attenuate broadband directional interference using an adaptive beamformer with very few number of adaptive filter coefficients [5]. One of the widely studied FIB is the uniform linear array (ULA) FIB [4-8] The ULA has a linear geometry with equal inter-sensor spacing. Due to this geometry, its angular resolution at boresight is better than that at its end-fire. In addition, this simple array structure enables many efficient direction-of-arrival (DOA) detection algorithms to be obtained. For example, the MUSIC algorithm [10] provides a high resolution method for detecting the angle of arrival (AoA) of the signal sources based on the subspace approach. The MUSIC algorithm can also detect wideband coherent sources in beamspace by performing MUSIC in beamspace using ULA-FIB [9]. Besides AoA estimation of wideband sources, adaptive interference suppression using beamspace adaptive beamforming [5] is very attractive because of the small number of adaptive weights required and the possibility of employing partial adaptation, yielding faster convergence and fewer number of high speed variable multipliers.

Given the advantages of ULA-FIB, electronic steerable uniform-circular arrays (UCAs) [1] with frequency invariant characteristics were studied in [14]. Unfortunately, the passband of a UCA is closely related to its radius and exhibit a bandpass characteristic. To obtain a frequency invariant characteristic over a large bandwidth, the dynamic range of the compensation filters will become very large and it leads to considerable noise amplification of the array. In this paper, we show that this problem can be overcome if uniform concentric circular arrays (UCCA) are employed. The sensors in a UCCA are placed on concentric circles with a uniform inter-sensor spacing and increasing radius. We find that UCAs with increasing radius will have their passbands moving towards the lower frequency bands. Hence, by using ring subarray with progressively larger radius in a UCCA, one can achieve a frequency invariant characteristic over a much larger bandwidth than a single UCA. Like UCAs, UCCAs differ from ULAs in the following: i) the azimuthal coverage of a UCCA is $360^{\circ}$ in contrast to $180^{\circ}$ of that in ULA, ii) the beampattern of UCCA is relatively uniform around the azimuth angle while that of ULA broadens as its beam is steered away from the boresight. As for DOA estimation, UCCA is suitable for 2D DOA estimation (azimuth and elevation angle) while the ULA is more suitable for azimuth angle DOA estimation [11]. Finally, as mentioned earlier, UCCA is able to form electronic steerable beam patterns that are relatively invariant with frequency over a wide bandwidth.

Similar to the FI UCAs in [14], the basic idea of the FI UCCA is to transform each snapshot sampled by the array to the phase modes via an Inverse Discrete Fourier Transform (IDFT). The transformed data is then filtered to compensate for the frequency dependence of the phase modes. Finally, these frequency invariant phase-modes are linear combined using a set of weights or coefficients to obtain the desired frequency invariant beam patterns. These weights, which govern the far field pattern of the UCCA, can be designed by conventional 1D digital filter design techniques such as the Parks-McClellan algorithm. Alternatively, different beam patterns can be created by varying these coefficients in an adaptive beamformer with approximately frequency invariant characteristics. The compensation filters are designed using second order cone programming (SOCP). Several design examples are given and the results show that electronic steerable beam patterns with approximately frequency invariant over a fairly large bandwidth can be obtained. This is very important in adaptive arrays where deep nulls in the target frequency band are desirable in suppressing broadband interference. To demonstrate some of the potential benefits of the FIB UCCA, a broadband DOA estimation using the UCCA beamformer is also given and the simulation result is very satisfactory. The paper is organized as follows: In section II, UCCA is introduced. In section III, the digital broadband UCCA FIB design is presented. In section IV, several design examples are given. In section $\mathrm{V}$, conclusions are drawn.

\section{UNIFORM CONCENTRIC CIRCULAR ARRAY (UCCA)}

Figure 1 shows a UCCA with $P$ rings and each ring has $K_{p}$ omnidirectional sensors located at $\left\{r_{p} \cos \phi_{k_{p}}, r_{p} \sin \phi_{k_{p}}\right\}$ (represented as Cartesian Coordinate with the center as the origin) where $r_{p}$ is the radius of the $p^{\text {th }}$ ring, $p=1, \cdots, P$, $\phi_{k_{p}}=2 \pi k_{p} / K_{p}$ and $k_{p}=0, \cdots, K_{p}-1$ as shown in Figure 2. In UCCAs, the inter-sensor spacing in each ring is fixed at $\lambda / 2$ where $\lambda$ is the smallest wavelength of the array to be operated and is denoted by $\lambda_{s}$. The radius of the $p^{\text {th }}$ ring of the UCCA is 
given by $\quad r_{p}=\lambda_{s} /\left(4 \sin \left(\pi / K_{p}\right)\right)$.

For convenience, this radius is represented as its normalized version $\quad \hat{r}_{p}=r_{p} / \lambda_{s}=1 /\left(4 \sin \left(\pi / K_{p}\right)\right)$.

Let $\alpha$ denote the ratio of the sampling frequency $f_{\mathrm{s}}$ to the maximum frequency $f_{\max }\left(\alpha=f_{s} / f_{\max }\right)$, the phase difference between the $k_{p}^{\text {th }}$ sensor and the center of the UCCA is $\chi_{k_{p}}=2 \pi \hat{r}_{p} \alpha \sin \theta \cos \left(\phi-\phi_{k_{p}}\right)$, and the corresponding phase shift is $e^{j \omega \hat{r}_{p} \alpha \sin \theta \cos \left(\phi-\phi_{k_{p}}\right)}$, where $\phi$, and $\theta$ are the azimuth angle and the elevation angle respectively, as shown in figure 3 . Hence, the steering vector [1] of the $p^{\text {th }}$ ring of a UCCA is: $\mathbf{s}=\left[e^{j \omega \hat{r}_{p} \alpha \sin \theta \cos \left(\phi-\phi_{0}\right)} e^{j \omega \hat{r}_{p} \alpha \sin \theta \cos \left(\phi-\phi_{1}\right)} \cdots e^{j \omega \hat{r}_{p} \alpha \sin \theta \cos \left(\phi-\phi_{K_{p}-1}\right)}\right]$.

The azimuth angle $\phi$ is on the horizontal plane where the sensors are situated. It measures from a reference imaginary axis on this horizontal plane, while the elevation angle $\theta$ is measured from a reference imaginary axis perpendicular to the horizontal plane. Without loss of generality, our design will be focused at an elevation angle of $\theta=\pi / 2$, i.e. the horizontal plane.

\section{DIGITAL BROADBAND UCCA FIB}

Figure 4 shows the structure of the broadband FIB for the $p^{\text {th }}$ ring of a UCCA. After appropriate down-converting, lowpass filtering and sampling, the sampled signals from the antennas are given by the vector $\mathbf{X}_{p}[n]=\left[x_{0}[n] x_{1}[n] \cdots x_{K_{p-1}}[n]\right]^{T}$, which is called a snapshot at sampling instance $n$. This snapshot is IDFT transformed to the phase-mode and the transformed snapshot is denoted by $\mathbf{V}_{p}[n]=\mathbf{W}_{M_{p} K_{p}} \cdot \mathbf{X}_{p}[n]$, where $\mathbf{W}_{M_{p} K_{p}}$ is an $M_{p}$ by $K_{p}$ IDFT matrix with $\left[\mathbf{W}_{M_{p} K_{p}}\right]_{m_{p}, k_{p}}=e^{j 2 \pi n_{p} k_{p} / K_{p}}$

and

$$
\left[\mathbf{V}_{p}[n]\right]_{m_{p}}=v_{m_{p}}[n]=\sum_{k_{p}=0}^{K_{p}-1} x_{k_{p}}[n] e^{j \frac{2 \pi k_{p} m_{p}}{K_{p}}}
$$

Here, $[\mathbf{A}]_{m, n}$ denotes the (mxn) entry of matrix $\mathbf{A}$. We assume that $M_{p}$ is an odd number and define $L_{p}=\left(M_{p}-1\right) / 2$. Each branch of the IDFT output is then filtered by $H_{m_{p}}(\omega)$ (to compensate for the frequency dependency as we shall see later in this section), multiplied with $g_{m_{p}}$ before combining to give the beamformer output $y_{p}[n]$ :

$$
y_{p}[n]=\sum_{m_{p}=-L_{p}}^{L_{p}}\left(v_{m_{p}}[n] * h_{m_{p}}[n]\right) \cdot g_{m_{p}},
$$

where $*$ denotes discrete-time convolution. To obtain the spatial-temporal transfer function of the beamformer, let us assume that there is only one source signal $s(n)$ with spectrum $S(\omega)$. Taking the Discrete Time Fourier Transform (DTFT) of equation (3), one gets

$$
V_{m_{p}}(\omega)=\sum_{k_{p}=0}^{K_{p}-1} X_{k_{p}}(\omega) e^{j \frac{2 \pi k_{p} m_{p}}{K_{p}}}=S(\omega) \sum_{k_{p}=0}^{K_{p}-1} e^{j \omega \hat{r}_{p} \alpha \cos \left(\phi-\phi_{k_{p}}\right)} e^{j \frac{2 \pi k_{p} m_{p}}{K_{p}}} .
$$

Taking DTFT on both size of equation (4) and using (5a), we have

$$
\begin{aligned}
& Y_{p}(\omega)=\sum_{m_{p}=-L_{p}}^{L_{p}} g_{m_{p}} V_{m_{p}}(\omega) H_{m_{p}}(\omega) \\
& =S(\omega) \sum_{m_{p}=-L_{p}}^{L_{p}} g_{m_{p}}\left(\sum_{k_{p}=0}^{K_{p}-1} e^{j \omega \hat{r}_{p} \alpha \cos \left(\phi-\phi_{k_{p}}\right)} e^{j \frac{2 . \alpha_{\alpha} m_{p}}{K_{p}}}\right) H_{m_{p}}(\omega)
\end{aligned}
$$

Hence, the spatial-temporal response of the $p^{\text {th }}$ ring is

$$
G_{p}(\omega, \phi)=\sum_{m_{p}=-L_{p}}^{L_{p}} g_{m_{p}}\left[\sum_{k_{p}=0}^{K_{p}-1} e^{j \omega \hat{r}_{p} \alpha \cos \left(\phi-\phi_{k_{p}}\right)} e^{j \frac{2 . \lambda_{p} m_{p}}{K_{p}}} H_{m_{p}}(\omega)\right] \text {. }
$$

To obtain a frequency invariant response, the term inside the bracket should be independent of the frequency variable $\omega$. First of all, using the expansion [13],

$$
e^{j \beta \cos \gamma}=\sum_{n=-\infty}^{+\infty} j^{n} J_{n}(\beta) e^{j n \gamma},
$$

where $J_{n}(\beta)$ is the Bessel function of the first kind, (6) can be rewritten as

$$
\begin{aligned}
G_{p}(\omega, \phi) & =\sum_{m=-L_{p}}^{L_{p}} g_{m_{p}} \sum_{k_{p}=0}^{K_{p}-1} \sum_{n=-\infty}^{+\infty} j^{n} J_{n}\left(\omega \hat{r}_{p} \alpha\right) e^{j n\left(\phi-2 n k_{p} / K_{p}\right)} e^{j \frac{2 \pi k_{p} m_{p}}{K_{p}}} H_{m_{p}}(\omega) \\
& =\sum_{m_{p}=-L_{p}}^{L_{p}} g_{m_{p}} H_{m_{p}}(\omega) \sum_{n=-\infty}^{+\infty} j^{n} J_{n}\left(\omega \hat{r}_{p} \alpha\right) e^{j n \phi}\left(\sum_{k_{p}=0}^{K_{p}-1} e^{j 2 \pi k_{p}\left(\frac{m_{p}-n}{K_{p}}\right)}\right)
\end{aligned}
$$

$\sum_{k_{p}=0}^{K_{p}-1} e^{j 2 \pi k_{p}\left(\frac{m_{p}-n}{K_{p}}\right)}=\left\{\begin{array}{cc}K_{p} & m_{p}-n=K q \\ 0 & \text { otherwise }\end{array}, \quad\right.$ where $\quad q \in \mathrm{Z}$.

Substituting (9) into (8) gives

$G_{p}(\omega, \phi)=K_{p} \sum_{m=-L_{p}}^{L_{p}} g_{m_{p}}\left[\sum_{n=m_{p}+K_{p} q} j^{n} J_{n}\left(\omega \hat{r}_{p} \alpha\right) e^{j n \phi}\right] H_{m_{p}}(\omega)$.

From [13], the Bessel function has the following property

$$
\left|J_{|n|}\left(\omega \hat{r}_{p} \alpha\right)\right| \leq\left(\frac{\omega \hat{r}_{p} \alpha e}{2|n|}\right)^{|n|} .
$$

Therefore, for sufficiently large value of $n$, the value of the Bessel function will be negligibly small. In other words, if the number of sensors is large enough, $G_{p}(\omega, \phi)$ can be approximated by

$G_{p}(\omega, \phi) \approx K_{p} \sum_{m_{p}=-L_{p}}^{L_{p}} g_{m_{p}}\left[j^{m_{p}} J_{m_{p}}\left(\omega \hat{r}_{p} \alpha\right) H_{m_{p}}(\omega)\right] \cdot e^{j m_{p} \phi}$.

It can be seen that for a given radius $r_{p}$, the bandwidth of the array, without compensation, is determined by the term $J_{m_{p}}\left(\omega \hat{r}_{p} \alpha\right)$. Rings with small radi usually have better high frequency response and vice versa. Therefore, to obtain a FI with large bandwidth, small responses of $J_{m_{p}}\left(\omega \hat{r}_{p} \alpha\right)$ at certain frequencies have to be compensated by $H_{m_{p}}(\omega)$. This is undesirable in general because it leads to considerable noise amplification. Fortunately, by employing more rings in a UCCA, a wider bandwidth can be obtained.

In a UCCA FIB, the outer rings have more phase modes than the inner ones. Let the weighting vectors of the rings be identical, i.e. $\mathbf{g}_{1}=\mathbf{g}_{2}=\cdots=\mathbf{g}_{P}$, where $g_{p}=\left[g_{-L_{P}} \cdots g_{L_{P}}\right]^{T}$. The overall response of the beamformer can be written as:

$$
\begin{aligned}
G(\omega, \phi) & =\sum_{p=1}^{P} G_{p}(\omega, \phi) \\
& =\sum_{m=-L_{P}}^{L_{P}} g_{m} \cdot e^{j m \phi} \cdot \sum_{p=1}^{P} K_{p}\left[j^{m} J_{m}\left(\omega \hat{r}_{p} \alpha\right) H_{m}^{p}(\omega)\right]
\end{aligned}
$$

where for notation convenience, we write $H_{m}^{p}(\omega)$ as $H_{m_{p}}(\omega)$. If the filters $H_{m}^{p}(\omega)$ are designed such that

$$
\sum_{p=1}^{P} K_{p}\left[j^{m} J_{m}\left(\omega \hat{r}_{p} \alpha\right) H_{m}^{p}(\omega)\right] \approx 1 \text { for } \omega \in\left[\omega_{L}, \omega_{U}\right],
$$

where $\omega_{L}$ and $\omega_{U}$ are respectively the lower and upper frequencies of interest, then the beamformer in (13) will be approximately frequency invariant within $\omega \in\left[\omega_{L}, \omega_{U}\right]$ and 


$$
G(\phi) \approx \sum_{m=-L_{P}}^{L_{P}} g_{m} e^{j m \phi} .
$$

Furthermore, its far field pattern is now governed by the spatial weighting $\left\{g_{m}\right\}$ alone. Since the right hand side of (14) is a linear function of the filter coefficients in $H_{m}^{p}(\omega)$ 's, the design problem in (14) can be treated as a filter design problem with all the filter outputs adding up to a desire response of value 1. If the minimax error criterion is used, the filter coefficients for $H_{m}^{p}(\omega)$ can be determined by second order programming (SOCP) [15]. It can also be seen from (15) that the far field spatial response is similar to that of a digital FIR filter with impulse response $\left\{g_{m}\right\}$. Therefore, $G(\phi)$ can be designed by conventional filter design algorithms such as the ParksMcClellan algorithm or SOCP if convex quadratic constraints are to be imposed. In addition, angular shifted versions of (15) can be derived by modulating $\left\{g_{m}\right\}$ with sinusoids at appropriate frequencies. For example, if the shift is $\pi / 2$, then the modulation is $\left\{e^{j \frac{\pi}{2} m}\right\}, m=-L_{P}, \ldots, L_{P}$. In example 3 to be described in Section IV, this property is used to generate a set of broadband beamformers uniformly spaced in the angular domain. Using a similar technique as in [9], DOA of broadband coherent signals can be estimated satisfactorily. Real-time adaptation of the beam pattern through the spatial weighting $\left\{g_{m}\right\}$ to suppress undesired interference is also simpler than traditional broadband adaptive array using tapped delay lines. Due to page limitation, detailed simulation results are omitted here. We now consider some design examples.

\section{DESIGN EXAMPLES}

\section{Example 1: UCCA-FIB with two rings.}

In this example, a two-ring UCCA is considered. The inner ring and the outer ring have 10 and 18 omnidirectional sensors, respectively. The required bandwidth of the UCCA-FIB is $\omega \in[0.15 \pi, 0.65 \pi]$. The numbers of phase modes $M$ are respectively 9 and 17 . We choose the central 9 spatial filter coefficients (phase mode) out of the 17 to shape the spatial response of the UCCA FIB. The desired beam is targeted at $60^{\circ}$ and the beamwidth is $10^{\circ} .\left\{g_{m}\right\}$ are obtained from the Parks-McClellan algorithm according to the given specification with same passband and stopband ripples. The frequency responses are shown in figures 5 and 6 . For convenience, the frequency responses of the UCCA-FIB for $\omega \in[0.15 \pi, 0.65 \pi]$ are overlapped together in figure 5 to illustrate the frequency invariant property of the beamformer. The frequency spectrum is approximately FI, with deep nulls formed at the desired position over the bandwidth of interest. Figure 6 shows the perspective view of the beamformer.

\section{Example 2: UCCA-FIB with three rings.}

A three-ring UCCA will be considered. The sensors in each ring are respectively 10,18 and 28 . The required FI bandwidth of the UCCA-FIB is $\omega \in[0.3 \pi, 0.75 \pi]$. The numbers of phase mode $M$ are 9,17 and 27 , respectively. The 17 spatial filter coefficients (phase mode) out of the 27 are used to shape the FIB-UCCA spatial response. The desired beam is targeted at $-20^{\circ}$ and the beamwidth is $10^{\circ}$. Again, $\left\{g_{m}\right\}$ is designed using the Parks McClellan algorithm with the given specification and identical passband and stopband ripples. The frequency responses of the FIB in the range $\omega \in[0.3 \pi, 0.75 \pi]$ are shown in figure 7 . The number of freedom and stopband attenuation are increased for the 3-ring case.

\section{Example 3: DOA Estimation using the UCCA beamformers}

In this example, the UCCA beamformer is used to find the DOAs of two arriving coherent signals at $35^{\circ}$ and $40^{\circ}$. The first signal is composed of 33 sinusoidal signals with frequencies ranging from $0.8 \times 10^{8}$ to $4 \times 10^{8}$ at an interval of $0.1 \times 10^{8} \mathrm{~Hz}$. The other signal is a 10 sample delayed version of the first signal and the sampling rate $\alpha$ is set to 2 . The basic beamformer is the same as the one in example 1, while the others are obtained by shifting its response equally in the angular domain. The SNR is $25 \mathrm{~dB}$ and the number of arrays used in the beamspace is 8 . The DOA estimation method used is the beam-space MUSIC method in [9], which was designed for FIB ULA. Figure 8 shows the MUSIC spectrum obtained in dB and the estimated angles are found to be $34.35^{\circ}$ and $39.59^{\circ}$, which are very close to the true values.

\section{CONClusion}

The theory and design of uniform concentric circular array (UCCA) having nearly frequency invariant (FI) characteristics are presented. By compensating the frequency dependency of individual phase modes using a digital beamforming network, the far field pattern of the array is determined by a set of weights and it is approximately invariant over a wide range of frequencies. Compared with FI uniform circular array (UCA), FI UCCA is able to achieve a wider bandwidth. The principle and usefulness of the UCCA-FIB in broadband DOA estimation are illustrated by design examples.

\section{REFERENCES}

[1] D. H. Johnson and D. E. Dudgeon, Array signal processing. concepts and techniques, Prentice Hall, 1993.

[2] H. Krim and M. Viberg, "Two decades of array signal processing research: the parametric approach," IEEE Signal Processing Mag., vol. 13, pp. 67-94, Jul. 1996.

[3] B. D. Van Veen and K. M. Buckley, "Beamforming: a versatile approach to spatial filtering," IEEE ASSP Mag., vol. 52, pp. 4-24, Apr. 1988.

[4] K. Nishikawa, T. Yamamoto, K. Oto and T. Kanamori, "Wideband beamforming using fan filter," in Proc. IEEE ISCAS, vol. 2., pp. 533-536, 1992.

[5] T. Sekiguchi and Y. Karasawa, "Wideband beamspace adaptive array utilizing FIR Fan filters for multibeam forming," IEEE Trans. Signal Processing, vol. 48, pp. 277-284, Jan. 2000.

[6] D. B. Ward, R. A. Kennedy and R. C. Williamson, "Theory and design of broadband sensor arrays with frequency invariant far-field beam patterns," J. Acoust. Soc. Amer., vol. 97, no.2, pp.1023-1034, Feb. 1995.

[7] D. B. Ward, R. A. Kennedy and R. C. Williamson, "FIR filter design for frequency invariant beamformers," IEEE Signal Processing Lett., vol.3, pp. 69-71, Mar. 2000.

[8] M. Ghavami and R. Kohno, "Recursive fan filters for a broad-band partially adaptive antenna," IEEE Trans. Commun., vol. 48, pp.185188, Feb. 2000.

[9] D. B. Ward, Z. Ding and R. A. Kennedy, "Broadband DOA estimation using frequency invariant beamforming," IEEE Trans. Signal Processing, vol. 46, pp. 1463-1469, May 1998.

[10] R. O. Schmidt, "Multiple emitter location and signal parameter estimation," IEEE Trans. Antennas Propagat., vol. AP-34, pp. 276280, Mar. 1986

[11] C. P. Mathews and M. D. Zoltowski, "Eigenstructure techniques for 2-D angle estimation with uniform circular arrays," IEEE Trans. Signal Processing, vol. 42, pp. 2395-2407, Sep. 1994.

[12] H. Steyskal, "Circular array with frequency-invariant pattern," Antennas and Propagation Society International Symposium 1989, AP-S. Digest, vol. 3, pg. 1477-1480, 1989.

[13] M. Abramowitz and I. A. Stegun, Handbook of Mathematical Functions, New York: Dover, 1965.

[14] S. C. Chan and Carson K. S. Pun, "On the design of digital broadband beamformer for uniform circular array with frequency 
invariant characteristics," IEEE ISCAS2002, vol. 1, pp. 693-696, May 2002.

[15] J. O. Coleman and D. P. Scholnik, "Design of nonlinear phase FIR Filters with second-order cone programming," in Proc. MWSCAS, vol. 1, pp. 409-412, 1999.

\section{FiguRES}

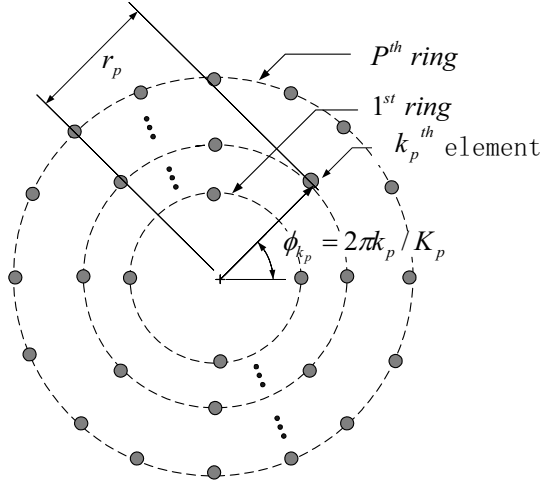

Figure 1. A UCCA with $P$ rings and $K_{p}$-sensor at each ring.

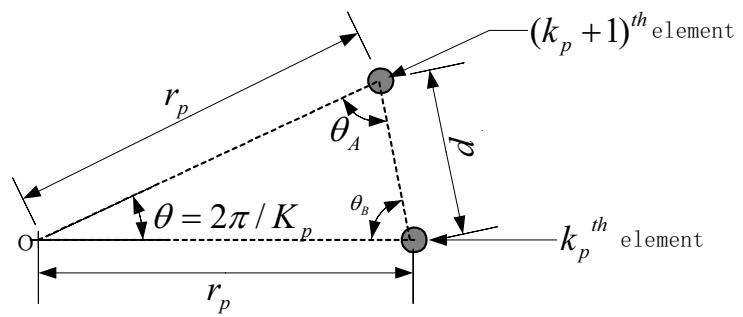

Figure 2. Relationship between inter-sensor spacing and the radius of the $p^{\text {th }}$ ring of the UCCA.

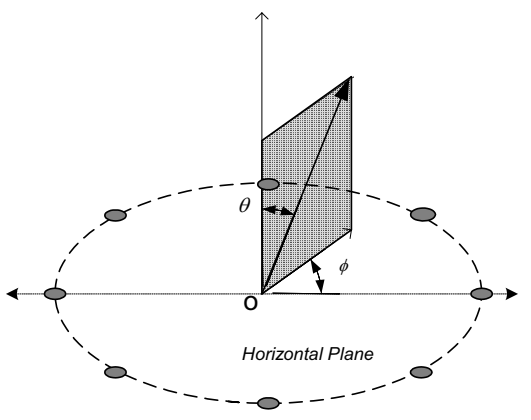

Figure 3. Geometry of the reference imaginary frame.

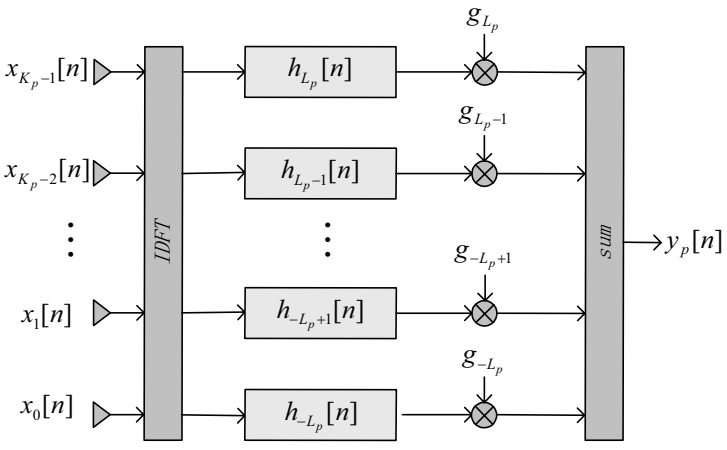

Figure 4. UCCA-FIB block diagram for the $p^{\text {th }}$ ring.

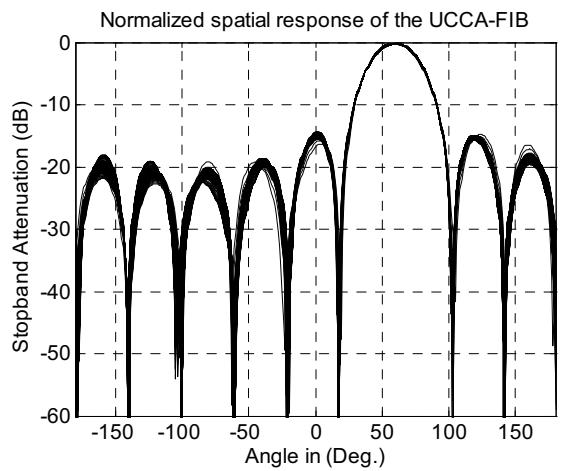

Figure 5. Spatial response of the UCCA-FIB with 2 rings.

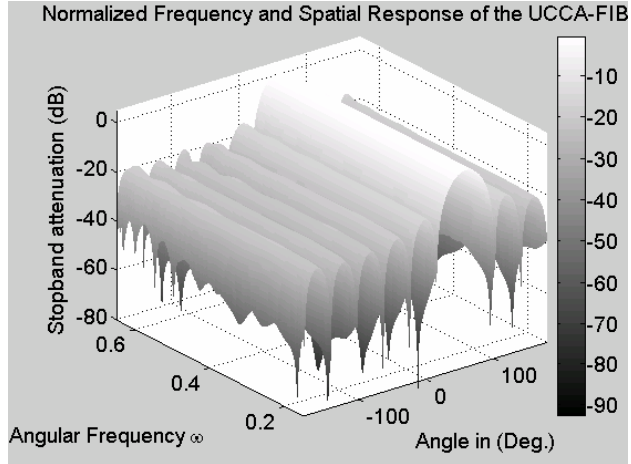

Figure 6. Spatial response and frequency response of the UCCA-FIB with 2 rings.

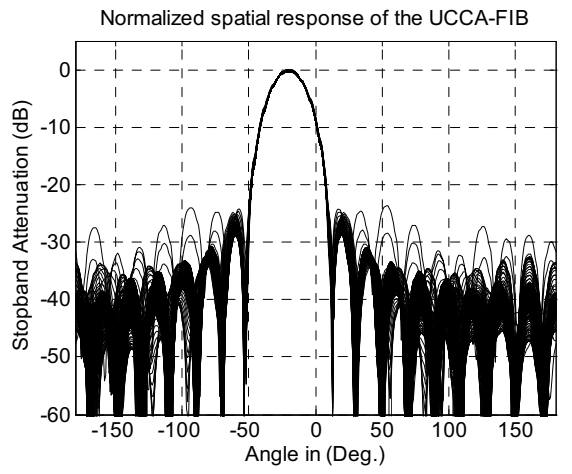

Figure 7. Spatial response of the UCCA-FIB with 3 rings.

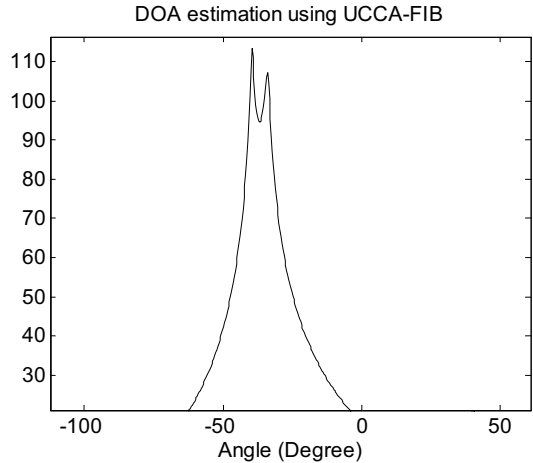

Figure 8 DOA estimation of two coherent sources based on the UCCAFIB with 2 rings. 